\title{
Correction to: Detection of freshwater mussels (Sinanodonta spp.) in artificial ponds through environmental DNA: a comparison with traditional hand collection methods
}

\author{
Daisuke Togaki ${ }^{1,2}$ (D) Hideyuki Doi ${ }^{3} \cdot$ Izumi Katano $^{1,4}$
}

Published online: 13 March 2020

(c) The Japanese Society of Limnology 2020

\section{Correction to: Limnology (2020) 21:59-65 \\ https://doi.org/10.1007/s10201-019-00584-0}

In the original publication of the article the sentence under the heading "Primer and probe design" was published incorrectly. The correct sentence is given in this correction.

To detect Sinanodonta DNA using real-time PCR, forward and reverse primers and a TaqMan probe for a 111-bp fragment of the $12 \mathrm{~S}$ region of mitochondrial DNA (mtDNA) were designed using Primer3Plus (http://www.bioinforma tics.nl/primer3plus). The primers and probe were visually selected from potential primers and probes by aligning sequences from the targeted species and related bivalve species while checking for sequence mismatches, especially at the $5^{\prime}$ or $3^{\prime}$ edges of the primers. The sequences of the real-time PCR primers and TaqMan probe were as follows: Forward primer, Sina_12S_F: 5'-ATACCCGAC TACTGT ATGGCT-3' Reverse primer, Sina_12S_R: 5'-ATTAAGCCACAG GATCCCCT-3' TaqMan probe, Sina_12S_probe: 5'-(FAM)-ACCTCA AACAATGTGGCG GTGCT-(TAMRA)-3'.

Daisuke Togaki

d_togaki@yahoo.co.jp

1 Graduate School of Human Science and Environment,

University of Hyogo, 1-1-12 Shinzaike-Honcho,

Himeji 670-0092, Japan

2 Graduate School of Science and Engineering, Ehime University, 2-5 Bunkyo-cho, Matsuyama, Ehime 790-8577, Japan

3 Graduate School of Simulation Studies, University of Hyogo, 7-1-28, Minatojima-minamimachi, Chuo-ku, Kobe 650-0047, Japan

4 Graduate School of Humanities and Sciences, Nara Women's University, Kitauoyahigashi-machi, Nara 630-8506, Japan 